\title{
Solid-Phase PCR in Microwells: Effects of Linker Length and Composition on Tethering, Hybridization, and Extension
}

BioTechniques 32:410-420 (February 2002)

\author{
A. Carmon, T.J. Vision, S.E. \\ Mitchell, T.W. Thannhauser, \\ U. Müller ${ }^{1}$, and S. Kresovich \\ Cornell University, Ithaca, and \\ ${ }^{1}$ Corning, Corning, NY, USA
}

\section{INTRODUCTION}

The solid-phase PCR (SP-PCR) (9) is analogous to standard PCR, except that one of the oligonucleotide primers, normally free to bind template DNA in solution, is attached to a surface (usually glass slides, beads, or plastic microwells). The potential uses of SP-PCR include genotyping, disease diagnostics, pathogen detection, and gene expression studies. During SP-PCR, a complementary single-stranded template anneals to the tethered oligonucleotide, DNA polymerase extends the molecule, the strands are denatured, and the cycle repeats as the liquidphase template concentration increases. After amplification is complete, untethered molecules are washed away, and the single- or double-stranded tethered extension products may be detected by a variety of means $(2,10,13)$.

Microplate-based solid-phase extension products are usually detected by enzymatic assays (8-11). For some applications, however, it would be preferable to employ direct detection of fluorescent products, which would allow quantitative estimation of yield over a wide dynamic range, as well as having the advantages of simplicity, flexibility, and cost. Thus, it would facilitate the use of microplate-based SP-PCR in high-throughput, automated applications. In our laboratory, however, SPPCR yields have not been sufficient for direct fluorescence detection with standard plate readers. We require approximately 100 fmol product in a $50 \mu \mathrm{L}$ volume/well for reliable quantification.

Previous studies have demonstrated that steric hindrance inhibits the hybridization of DNA in solution to im- mobilized oligonucleotides $(6,12)$. Steric hindrance might also affect solid-phase polymerization by impeding the attachment of Taq DNA polymerase to tethered oligonucleotides that directly abut the supporting surface. It has been demonstrated that SP-PCR efficiency is enhanced when a polydeoxythymidine (dT) spacer is included at the $5^{\prime}$ end of the solid-phase primer $(1,10,13)$. Solid-phase oligonucleotides containing $5^{\prime}(\mathrm{dT})_{n}$ spacers are desirable because they are inexpensive and easy to synthesize. However, we tend to observe high background signals when using these primers to amplify AT-rich plant DNA templates.

In this study, we quantitatively measured the outcome of tethering, hybridization, and solid-phase extension in microwells using common fluorescent labels and a standard plate reader. We also evaluated the effect of $5^{\prime}$ hexaethyleneglycol (HEG) spacers of varying lengths in mitigating steric hindrance to hybridization versus polymerization, and compared the performance of solid-phase primers with 5-unit HEG and (dT) 10 spacers (both type spacers were coupled to a $5^{\prime}$ amino modifier C6 residue). The goals were to demonstrate that SP-PCR was occurring in microwells and to develop a SPPCR protocol suitable for use in quantitative assays that employ direct fluorescence detection in plate format.

\section{MATERIALS AND METHODS}

\section{Oligonucleotides}

Primers and probes (Table 1) were derived from the first exon of the Ara- 


\section{Research Report}

Table 1. Oligonucleotides Used for Evaluation of Solid-Phase Extension and PCR

\begin{tabular}{|c|c|c|c|}
\hline Oligonucleotide & $\begin{array}{c}5^{\prime} \\
\text { Modification }\end{array}$ & $\begin{array}{l}\text { DNA Sequence } \\
\qquad\left(5^{\prime} \rightarrow 3^{\prime}\right)^{\mathrm{a}}\end{array}$ & $\begin{array}{l}\text { Experimental } \\
\text { Role }\end{array}$ \\
\hline $\mathrm{F}^{(\mathrm{HEG})}{ }_{\mathrm{n}}$ & aminoC6b-(HEG)c & GCCTTTTTATGCGATTCTGC & Tethered in microwells \\
\hline$F(d T)_{10}$ & aminoC6 & TTTTTTTTTTGCCTTTTTATGCGATTCTGC & Tethered in microwells \\
\hline $80^{f l}$ & fluorescein & $\begin{array}{l}\text { CAGGCACCTCATCAGGACTCACAGGATCCAAATCTA } \\
\text { TAACAAGACCTTCCTCAATE्CGGTGCAGAATCGCAT } \\
\text { AAAAAGGC }\end{array}$ & $\begin{array}{l}\text { Hybridized to tethered } \\
\text { primers (solid-phase } \\
\text { extension assay) }\end{array}$ \\
\hline $\mathrm{F}$ & none & GCCTTTTTATGCGATTCTGC & $\begin{array}{l}\text { Liquid-phase primer } \\
\text { (SP-PCR) }\end{array}$ \\
\hline $\mathrm{R}^{f l}$ & fluorescein & CGGGTAGGAGTACCTTGAAT & $\begin{array}{l}\text { Liquid-phase primer } \\
\text { (SP-PCR) }\end{array}$ \\
\hline $\mathrm{R}^{\mathrm{tr}}$ & Texas Red & CGGGTAGGAGTACCTTGAAT & Probe (SP-PCR) \\
\hline \multicolumn{4}{|c|}{$\begin{array}{l}\text { a80fl contains a Hpall site (underlined) not found in the Arabidopsis genomic sequences. } \\
\text { bAmino modifier C6 (Glen Research, Sterling, VA, USA). } \\
\text { c0-20 molecules of hexaethyleneglycol (Spacer Phosphoramidite 18, Glen Research) }\end{array}$} \\
\hline
\end{tabular}

bidopsis thaliana phytochrome C $(P h y C)$ gene (4). Figure 1 shows the relative positions of oligonucleotides within $P h y C$.

\section{Covalent Binding of \\ Oligonucleotides to Wells}

In all experiments, 5' amino-modified oligonucleotides were tethered in

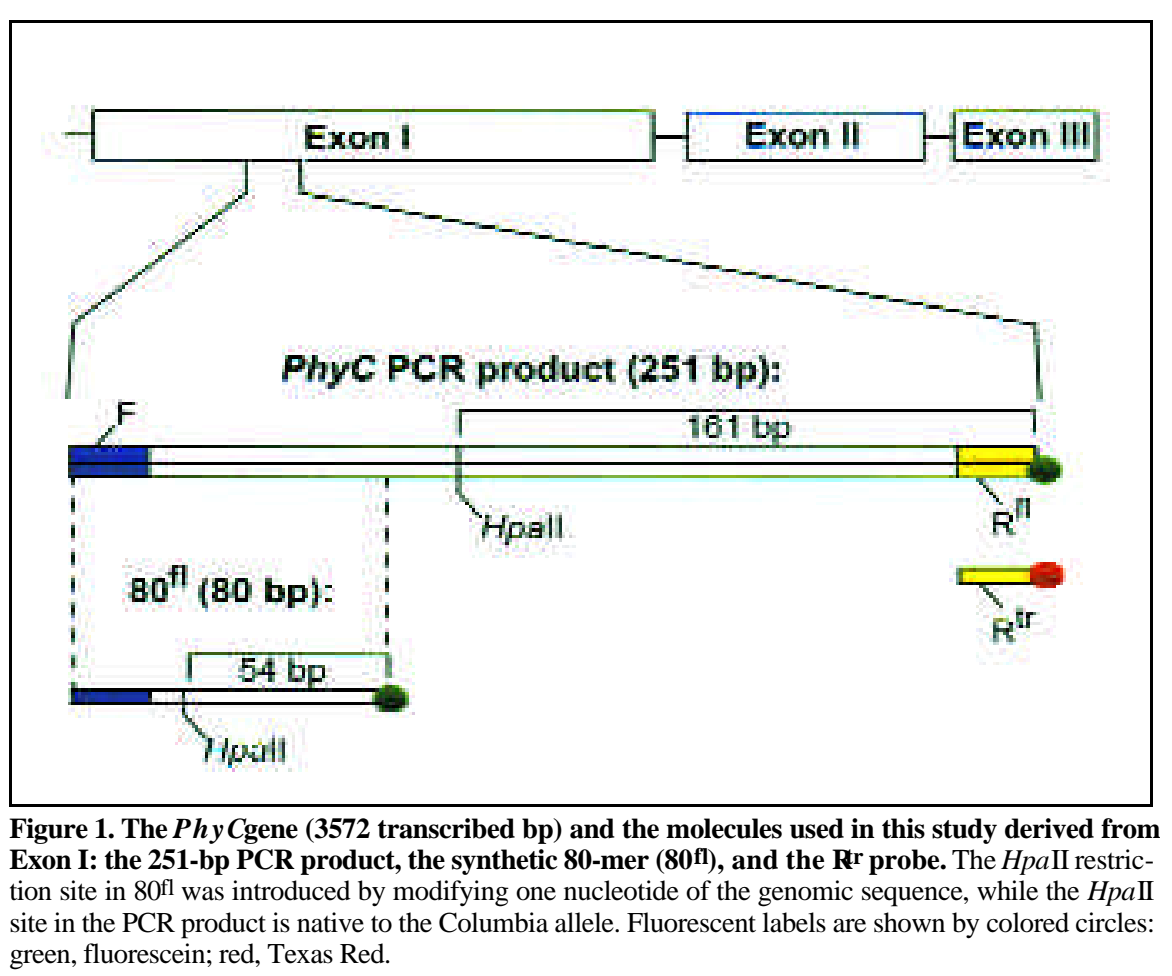

Figure 1. The Phy Cgene ( 3572 transcribed bp) and the molecules used in this study derived from Exon I: the 251-bp PCR product, the synthetic 80-mer $\left(\mathbf{8 0}^{\mathrm{fl}}\right)$, and the $\mathbf{R}^{\mathrm{tr}}$ probe. The HpaII restriction site in $80^{\mathrm{fl}}$ was introduced by modifying one nucleotide of the genomic sequence, while the HpaII site in the PCR product is native to the Columbia allele. Fluorescent labels are shown by colored circles: green, fluorescein; red, Texas Red.

NucleoLink ${ }^{\mathrm{TM}}$ (Nalge Nunc International, Rochester, NY, USA) strips (eight wells/strip) by standard carbodiimide-mediated condensation chemistry (10). Oligonucleotide tethering and blocking of unreacted primer binding sites followed the microwell manufacturer's protocol (http://nunc. nalgenunc.com/resource/technical/nag/ DP0063.htm).

\section{Optimization of Spacer Length}

Optimal spacer length was determined as outlined in Figure 2. Initially the $\mathrm{F}^{(\mathrm{HEG})}{ }_{n}$ oligonucleotides with spacer lengths of $0,5,10$, and 20 units were evaluated. Four trials (repetitions) were performed with eight 8-well strips/trial, and two wells/treatment/strip. Placement of treatments within strips was randomized. After tethering, the amount of covalently bound primer/ well was determined for one strip/trial using YOYO-1 iodide (Molecular Probes, Eugene, OR, USA), a fluorescent dye that has a strong affinity for ssDNA. Following the published protocol (7), fluorescence was measured with a SPECTRAFluor Plus plate reader (TECAN, Research Triangle Park, NC, USA). After initial results were obtained, a second experiment was performed in which spacers containing from 1-8 HEG residues were evaluated. Here, each spacer length was assigned to one well/strip, but as before, eight strips were used (seven experimental strips and one strip for quantification of tethered oligonucleotide).

The $80^{\mathrm{fl}}$ oligonucleotide $(5 \mathrm{pmol})$ was hybridized to tethered oligonucleotides in $100 \mu \mathrm{L} 5 \times \operatorname{SSC}(1.25 \mathrm{M}$ $\mathrm{NaCl}, 0.125 \mathrm{M}$ sodium citrate, $\mathrm{pH}$ 7.0) for $16 \mathrm{~h}$ at $50^{\circ} \mathrm{C}$. Wells were washed three times with $1 \times \mathrm{SSC}$ at room temperature to remove unhybridized 80 mer, $100 \mu \mathrm{L} 1 \times \mathrm{SSC}$ were added to 
each well, and the amount of fluorescein/well was determined using the plate reader. Tethered oligonucleotides $\left(\mathrm{F}^{(\mathrm{HEG})}{ }_{\mathrm{n}}\right)$ were extended in $50-\mu \mathrm{L}$ reaction volumes containing $2.5 \mathrm{mM}$ $\mathrm{MgCl}_{2}, 0.2 \mathrm{mM}$ each dNTP, and $2.5 \mathrm{U}$ Taq DNA polymerase in $1 \times$ PCR buffer (Promega, Madison, WI, USA). Reactions were incubated for $1 \mathrm{~h}$ at $50^{\circ} \mathrm{C}$, and wells were washed three times with $1 \times$ SSC. Restriction digests were done in $50-\mu \mathrm{L}$ volumes with $1 \times$ One-PhorAll Buffer PLUS (Amersham Biosciences, Piscataway, NJ, USA), 0.10 $\mathrm{mg} / \mathrm{mL}$ BSA (New England Biolabs, Beverly, MA, USA), and $1 \mathrm{U}$ HpaII (Invitrogen, Carlsbad, CA, USA). Reactions were incubated for $1 \mathrm{~h}$ at $37^{\circ} \mathrm{C}$. The reaction mixture $(40 \mu \mathrm{L})$ was then transferred to 96-well black plates (Corning Costar, Cambridge, MA, USA), a $60-\mu \mathrm{L}$ aliquot of TE $(10 \mathrm{mM}$ Tris-HCl, pH 8.0, 1 mM EDTA) was added, and fluorescence was measured.

Fluorescein-labeled restriction fragments were purified with Centri-Sep ${ }^{\mathrm{TM}}$ spin columns (Princeton Separations, Adelphia, NJ, USA). Samples were concentrated, and approximately onefourth of the original reaction volume was assayed on a model 377 automated DNA fragment analyzer using established protocols (GeneScan ${ }^{\circledR}$ Reference Guide; both from Applied Biosystems, Foster City, CA, USA).

\section{Quantification of SP-PCR Products}

Experiments for confirmation and quantification of SP-PCR products are diagrammed in Figure 3. Three trials were performed with four 8-well strips/trial and one treatment/strip. The presence of Taq DNA polymerase and tethered oligonucleotides were varied in each treatment, and one well/strip was reserved for quantification of tethered oligonucleotides with YOYO-1.

Total genomic DNA was extracted from A. thaliana cv. Columbia seedlings using a standard method (5). $\left.\mathrm{F}^{(\mathrm{HEG})}\right)_{5}$ oligonucleotides (5-unit spacers) were tethered as described. SPPCR buffers were as above, except that they contained 1 pmol F (unlabeled), 8 pmol $\mathrm{R}^{\mathrm{fl}}$ (5'-fluorescein) primers, and $25 \mathrm{ng}$ Arabidopsis genomic DNA. PCR was performed using a Primus 96-plus thermal cycler (MWG Biotech, Ebers-

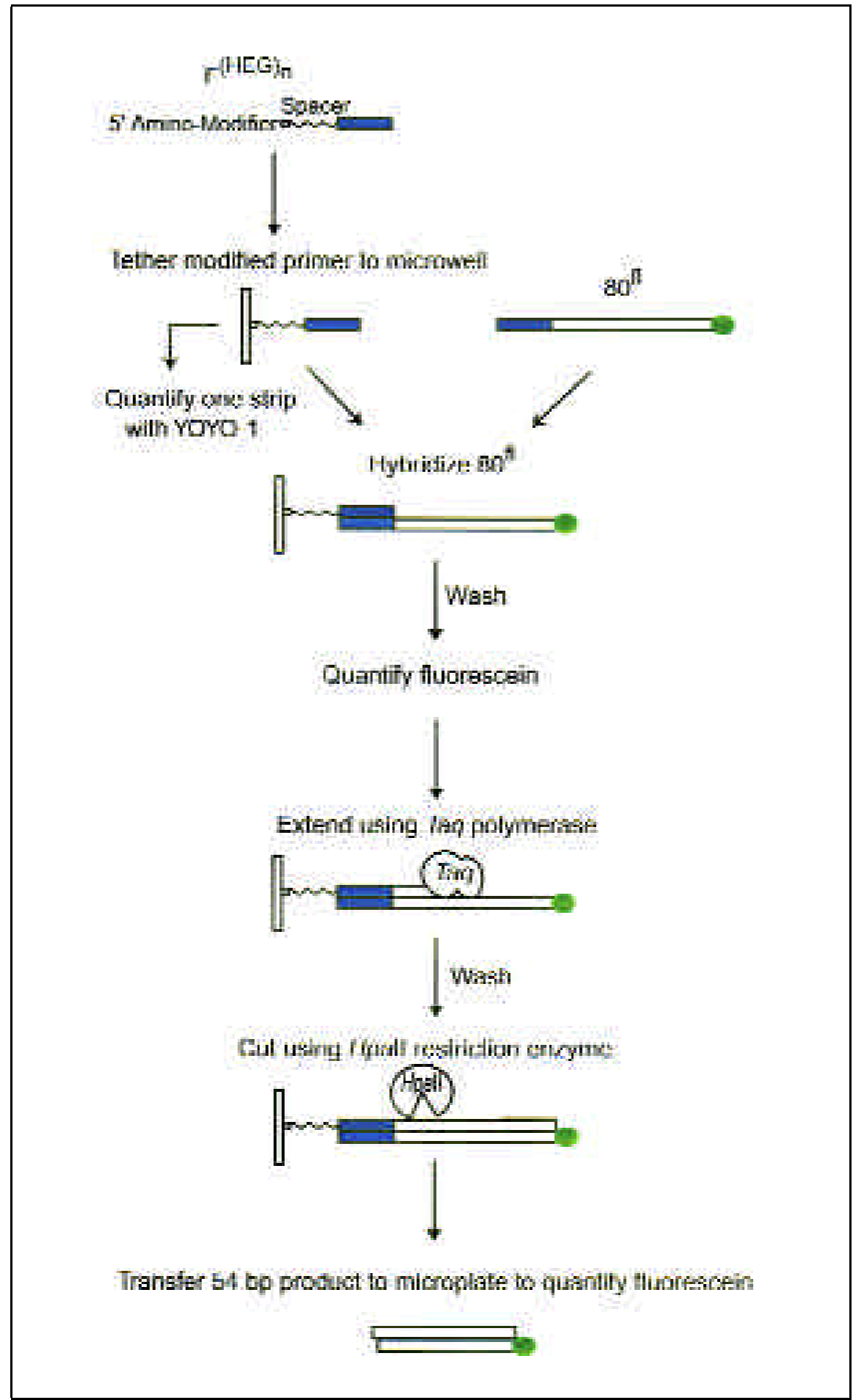

Figure 2. Hybridization and extension assay for determining optimal spacer length for tethered oligonucleotides. Experiment consisted of four trials with eight 8-well strips/trial and two wells/treatment/strip with random placement of treatments within strips. The amount of covalently bound primer was determined for one strip/trial using YOYO-1 iodide. 5' amino-modified primers with HEG spacers of varying lengths $\left[\mathrm{F}^{(\mathrm{HEG})}{ }_{\mathrm{n}}\right]$ were tethered to microwells. The amount of primer tethered in one 8-well strip/trial was determined by YOYO-1 binding. For seven strips, wells were hybridized to a fluoresceinlabeled synthetic 80 -mer $\left(80^{\mathrm{fl}}\right)$. Unhybridized $80^{\mathrm{fl}}$ was removed by washing, the quantity of remaining $80^{\mathrm{fl}}$ was measured, and the tethered primers were extended by Taq DNA polymerase. Double-stranded solid-phase extension products were digested with HpaII, and the liquid-phase fragments were then transferred to a microplate and quantified. For selected wells, samples were concentrated, and the presence of the expected 54-bp restriction fragment was confirmed on a DNA fragment analyzer. 
berg, Germany) with the following temperature profile: $95^{\circ} \mathrm{C}$ for $5 \mathrm{~min}, 35$ cycles of $95^{\circ} \mathrm{C}$ for $1 \mathrm{~min}, 55^{\circ} \mathrm{C}$ for 1 $\min , 72^{\circ} \mathrm{C}$ for $2 \mathrm{~min}$, followed by a 90 min incubation at $50^{\circ} \mathrm{C}$. To confirm that liquid-phase PCRs were successful, $10 \mu \mathrm{L}$ of the amplification reactions were run on a $1 \%$ agarose gel stained with ethidium bromide. For selected wells, HpaII digests were performed, and products were sized on a DNA fragment analyzer, as before, to confirm the presence of the expected 161-bp fragment (Figures 1 and 3A).

Quantitative estimates of SP-PCR yield were made as follows. After com pletion of SP-PCR, wells were washed three times with $1 \times \mathrm{SSC}$, and the amount of bound fluorescein signal was determined. Then, the dsDNA was denaturated by heating to $95^{\circ} \mathrm{C}$ for $5 \mathrm{~min}$, the solution was aspirated, the wells were washed three times with $1 \times \mathrm{SSC}$, and fluorescein readings were obtained to measure the residual fluorescein-labeled complementary template. The tethered DNA strands were then probed with $\mathrm{R}^{\operatorname{tr}}$ (Texas Red ${ }^{\circledR}$-labeled) oligonucleotide ( $5 \mathrm{pmol}$ in $50 \mu \mathrm{L} 5 \times \mathrm{SSC}$ ) for $16 \mathrm{~h}$ at $50^{\circ} \mathrm{C}$. Wells were washed, as before, and measurements of both hybridized $\mathrm{R}^{\mathrm{tr}}$ and residual fluorescein

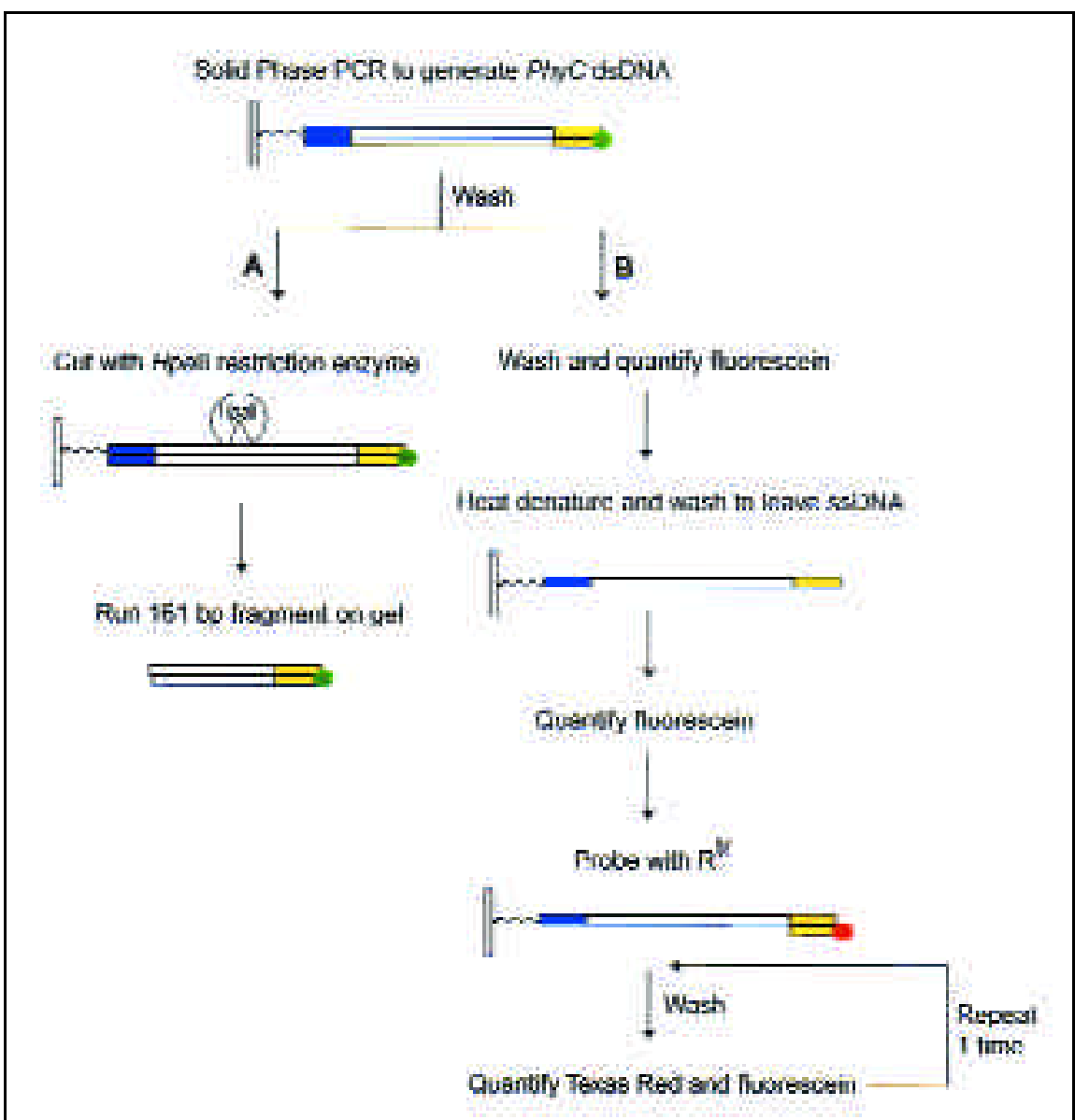

Figure 3. Experiments for verification and quantification of SP-PCR. Experiment consisted of three trials with four 8-well strips/trial and one treatment/strip. The presence of Taq DNA polymerase and tethered oligonucleotides were varied in each treatment, and the amount of covalently bound primer was determined for one well/strip using YOYO-1. Fluorescein-labeled double-stranded products were generated by inclusion of liquid-phase $\mathrm{R}^{\mathrm{fl}}$ primer. (A) Solid-phase extension was confirmed in selected wells by HpaII digestion of tethered dsDNA and visualization of the resulting 161-bp fragment on a denaturing polyacrylamide gel. (B) Solid-phase extension was quantified by denaturation of tethered double-stranded products, washing, and hybridization of a fluorescent probe $\left(\mathrm{R}^{\mathrm{r}}\right)$ complementary to the $3^{\prime}$ end of the single-stranded product. Wells were washed, and fluorescence was quantified by comparison to a standard. The amount of fluorescein-labeled complementary strand (and/or R liquid-phase primer) was also determined after completion of SP-PCR, denaturation, probing, and additional washing. 


\section{Research Report}

Table 2. SP-PCR Yields from Various Treatments Expressed as Mean fmol Fluorescein and Texas Red

\begin{tabular}{|c|c|c|c|c|c|c|c|}
\hline \multicolumn{2}{|c|}{ Treatmenta } & \multirow{2}{*}{$\begin{array}{c}\text { After PCR } \\
\text { Fluoresceinc }\end{array}$} & \multirow{2}{*}{$\begin{array}{l}\text { After Denaturation } \\
\text { Fluorescein }\end{array}$} & \multicolumn{2}{|c|}{ After Rr Hybridization } & \multicolumn{2}{|c|}{ After Further Washes } \\
\hline Taq & Tetherb & & & Fluorescein & Texas Redd & Fluorescein & Texas Red \\
\hline- & - & $25(6)^{e}$ & $21(6)$ & $6(8)$ & $91(10)$ & $7(7)$ & $94 \quad(6)$ \\
\hline- & + & $17 \quad(5)$ & $24(5)$ & $4(5)$ & $73 \quad(5)$ & $6(7)$ & $72 \quad(5)$ \\
\hline+ & - & 33 (8) & $26(5)$ & $2(5)$ & $53 \quad(5)$ & $3(4)$ & $50 \quad(5)$ \\
\hline+ & + & $124(10)$ & $29(7)$ & $21(6)$ & $263(25)$ & $14(5)$ & 252 (23) \\
\hline \multicolumn{8}{|c|}{$\begin{array}{l}\text { a Experiment consisted of three trials with four 8-well strips per trial and one treatment per strip. } \\
\left.b^{(} F^{(H E G)}\right)_{5} \text { was tethered in micorwells. }\end{array}$} \\
\hline
\end{tabular}

were taken. Fluorescence was determined one final time for both dyes after three additional washes with $1 \times$ SSC.

\section{Comparison of $5^{\prime}$ HEG and $\mathrm{dT}_{\mathbf{1 0}}$ Spacers}

$\mathrm{F}(\mathrm{HEG})_{5}$ and $\mathrm{F}^{(\mathrm{dT})} 10$ were each tethered to all eight wells of three NucleoLink strips apiece, and SP-PCRs were then performed as described above. In addition, there were three control strips that contained all reaction components except tethered oligonucleotides. Wells were washed, probed with $\mathrm{R}^{\mathrm{tr}}$, and fluorescence was measured as described above. The quantity of tethered oligonucleotide was determined by YOYO-1 assay for one well/strip.

\section{Statistical Analyses}

ANOVA was performed on fluorescence data using the JMP statistical software package (version 3.0; SAS Institute, Cary, NC, USA). Box-Cox transformations were used to obtain normally distributed residuals (3). Linear contrast tests (14) were used for planned comparisons among specific treatments. Variance components were estimated by equating observed to expected mean squares (14).

\section{RESULTS AND DISCUSSION}

\section{Optimization of 5' HEG Spacer Length}

We first sought to determine the op- timum length of $5^{\prime}$ HEG spacers on tethered primers for hybridization and extension. This experiment (Figure 2) was designed so that hybridization could be measured independent of solid-phase extension.

Previous reports based on enzymatic assays indicated that well-to-well variability was low for the NucleoLink surface (10). However, our ANOVA results indicated that most of the variation in hybridization and extension experiments $(65 \%$ and $83 \%$, respectively, of the experiment-wide variance) was due to inherent differences between wells of the same strip (likely due to variability in manufacture). By contrast, there was little variability among strips (4\% for hybridization and $8 \%$ for extension experiments, based on average values for eight wells/strip) and trials (31\% and 9\%, respectively).

The hybridization and extension results for each spacer length are shown in Figure 4. To establish a range for more detailed study, spacers with 0,5 , 10 , and 20 HEG residues were initially evaluated. While the amount of hybridized 80-mer decreased as a function of spacer length, solid-phase primer extension increased from 0 to 5 unit spacers, was roughly equivalent for 5- and 10-unit spacers, and decreased at 20-unit spacers (Figure 4, A and B). Thus, the comparatively inefficient extension of tethered primers without a $5^{\prime}$ spacer appears to be due to steric hindrance of Taq DNA polymerase rather than to lowered hybridization efficiency. Conversely, the decline in primer extension with 20-unit spacers might be related to decreased hybridization of the 80-mer (Figure 4A). The optimal spacer length for efficient solid-phase extension by Taq DNA polymerase appears to be 5-10 HEG units. When we evaluated spacers between one and eight HEG units in length, the results indicated a slight decline in hybridization with increasing spacer length and an increase in the efficiency of solidphase extension, with little difference in overall yield between five to eight linkers (Figure 4, C and D). Since shorter spacers are easier and less expensive to synthesize, subsequent SPPCR experiments were performed using tethered primers with 5 units of HEG spacer.

We should note that the decrease in hybridization yield observed with increasing spacer length was surprising. It could be argued that this result was due to fluorescence quenching, as increased concentrations of labeled oligomers were hybridized to tethered primers. Because we do not know the spatial distribution of hybridized fluorescein-labeled oligomers on the well surface, we cannot disregard the possibility that quenching might occur. However, our standard concentration curves for fluorescein in solution were linear $\left(\mathrm{R}^{2}=0.99\right)$ at $1 \mathrm{nmol}$ dye $/ 50 \mu \mathrm{L}$, about 2000-fold greater than the quantities of fluorescein detected in the SPPCR experiments. We should also mention that other researchers have observed decreased hybridization yields for long spacers relative to their shorter counterparts using radioactive hybridization assays. For three different 
Table 3. Quantification of Tethered Primer and Extended Solid-Phase Product with Spacers of Different Composition Expressed in Mean fmol Dye ${ }^{\mathrm{a}}$

\begin{tabular}{|c|c|c|}
\hline $\begin{array}{c}5^{\prime} \\
\text { Spacer }\end{array}$ & $\begin{array}{l}\text { Tethered Oligonucleotide } \\
\text { fmol YOYO-1 }\end{array}$ & $\begin{array}{l}\text { Product Extended } \\
\text { fmol Texas Red }\end{array}$ \\
\hline$(\mathrm{HEG})_{5}$ & $744(81)^{b}$ & $228 \quad(8)$ \\
\hline$(\mathrm{dT})_{10}$ & 713 (23) & $147 \quad$ (3) \\
\hline Controlc & $55 \quad(7)$ & $52 \quad(2)$ \\
\hline \multicolumn{3}{|c|}{$\begin{array}{l}\text { aExperiment consisted of three 8-well strips per spacer with one treatment per } \\
\text { strip. YOYO-1 was quantified in one well per strip. } \\
\text { b2 } 2 \text { standard error is shown in parentheses. } \\
\text { cControl wells contained all reactants except tethered primers. }\end{array}$} \\
\hline
\end{tabular}

glycol spacers (propanediol, diethyleneglycol, and triethyleneglycol), a steady increase in duplex yield was reported with increasing spacer length up to 8-10 units, but hybridization declined with further length increases until, at 25-30 units, duplex yield equaled that found with no spacer at all (12).

After hybridization of the 5' fluorescein-labeled 80-mer and extension of tethered primers (Figure 2), fragments detected after digestion with HpaII should represent double-stranded ex- tension products, since $\mathrm{HpaII}$ does not cut ssDNA. To verify that the observed fluorescence was associated with the appropriate restriction fragment and not residual, uncut 80-mer, aliquots from selected wells were loaded on a DNA fragment analyzer. There were intense fluorescent signals around 54 bp, the size of the expected HpaII restriction fragment, and no fluorescence in the 80-bp region (data not shown). Therefore, HpaII activity was either not affected by steric hindrance or the re-

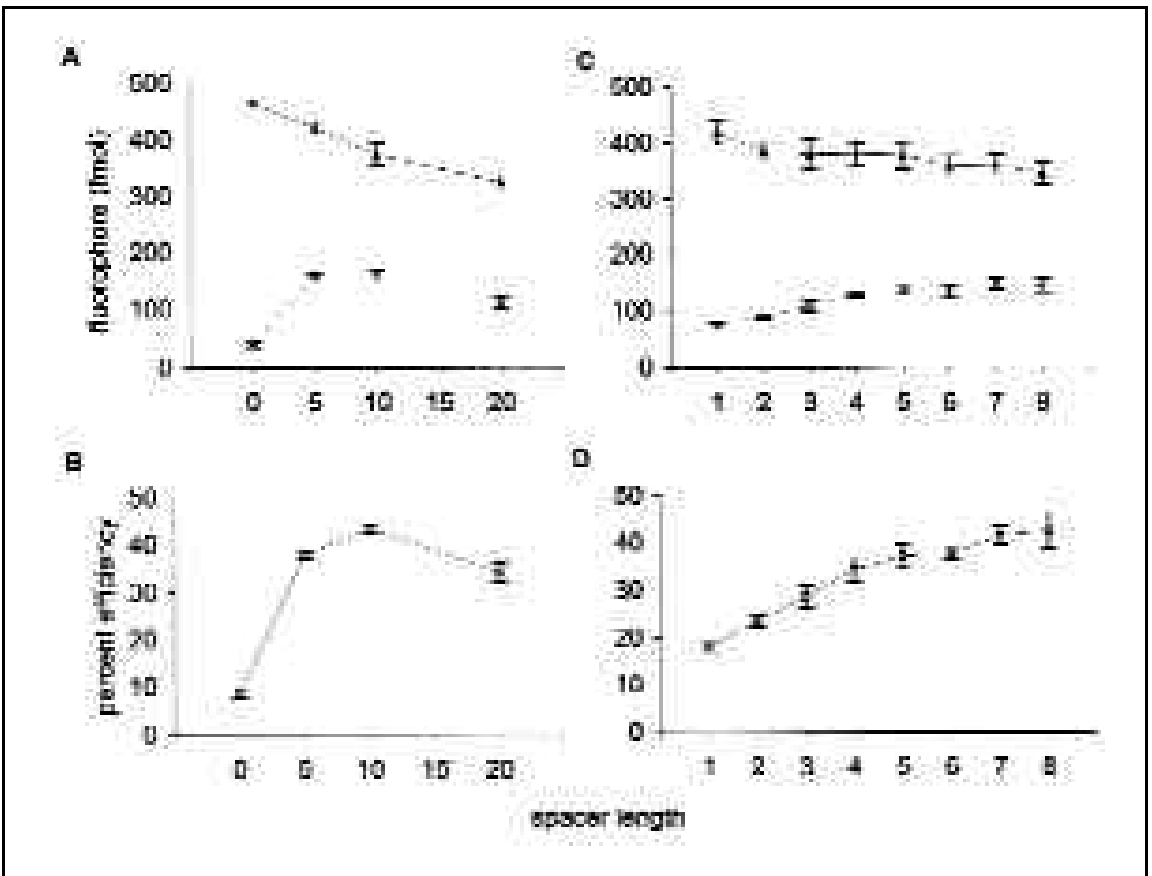

Figure 4. Hybridization and solid-phase extension of tethered oligonucleotides with $5^{\prime}$ HEG spacers of various lengths. Error bars represent $2 \times$ standard errors. (A) Quantity of fluorescent label (in fmol) after hybridization of $80^{\mathrm{fl}}$ to tethered primers (solid line) and after primer extension (dashed line), the latter measured as the quantity of labeled liquid-phase restriction fragment. Results are shown for spacers with $0,5,10$, and 20 HEG residues. (B) Percent efficiency of extension (extension $\times 100 /$ hybridization) shown in panel A. (C) Quantity of probe hybridized (solid line) and extended (dashed line) for spacers with 1-8 HEG residues, as in panel A. (D) Efficiency of extension for reactions shown in panel C. 
striction enzyme excess (approximately 140-fold) compensated for possible steric constraints.

\section{SP-PCR Verification and Quantification}

The previous experiments demonstrated solid-phase extension in the presence of abundant template. To test for solid-phase extension coupled with template amplification, two experiments were performed (Figure 3). SPPCRs were done using primers that am plify a 251-bp fragment from exon I of the A. thaliana PhyC gene (Figure 1). The 5 '-aminated $F^{(H E G)}{ }_{5}$ oligonucleotides were tethered, and PCRs were performed using a liquid-phase primer ratio of $1: 8\left(\mathrm{~F}: \mathrm{R}^{\mathrm{fl}}\right)$ to produce an excess of template strands complementary to the tethered oligonucleotide $(10,11)$.

Visualization of appropriately sized (161 bp) fluorescent restriction fragments confirmed the presence of SPPCR products in wells containing both tethered primers and Taq DNA polymerase (Figure 5A). Some but not all experimental wells (those with tethered primers and Taq) and some controls (those with Taq but without tethered primers) also contained a small amount of full-length, presumably residual single-stranded liquid-phase product (251 bp) that was not removed from wells by washing (Figure 5B).

Table 2 presents fluorescence data from the experiment outlined in Figure 3B. Statistical analyses indicated that there was a highly significant interaction term between the presence of Taq DNA polymerase and the presence of tethered primers $\left(P=6 \times 10^{-4}\right)$, and a linear contrast between the treatment having all SP-PCR components and the other three treatments revealed that the difference in fluorescence was highly significant $\left(P=3 \times 10^{-4}\right)$.

In this experiment (Figure 3B), fluorescein was quantified after completion of SP-PCR and after subsequent washings and hybridizations. The fluorescein signal represented either specific binding of unincorporated $\mathrm{R}^{\mathrm{fl}}$ liquidphase primers and/or fluorescein-labeled complementary PCR products to extended primers or nonspecific background. After completion of SP-PCR, approximately $100 \mathrm{fmol}$ fluorescein were detected in wells containing all reaction components (Table 2). Fluorescein signal dropped to background after heat denaturation, indicating that the fluorescein-labeled complements/primers were removed from wells. The quantity of solid-phase oligonucleotides extended during PCR was estimated by hybridization to $\mathrm{R}^{\mathrm{tr}}$ (Texas Redlabeled probe). Although background fluorescence was relatively high in the controls, the Texas Red signal from wells containing all SP-PCR components was approximately 3 -fold greater. After correction for background fluorescence in the Texas Red data (Table 2 ), we estimate that approximately 180 fmol tethered primers were extended by SP-PCR. This is consistent with the values obtained in earlier hybridization/extension experiments where approximately $160 \mathrm{fmol}$ product were detected for tethered oligonucleotides with 5unit HEG spacers (Figure 4A).

The Texas Red background remained after three additional washes with $1 \times \mathrm{SSC}$ at room temperature (Table 2). Among the controls, the highest background readings were in wells containing neither Taq DNA polymerase nor tethered oligonucleotides. However, the fluorescein readings in these wells were low, even immediately following completion of temperature cycling. Since the $\mathrm{R}^{\mathrm{tr}}$ probe appears to be interacting directly with the well surface, additional blocking steps, different blocking solutions, shorter hybridizations, or an alternative dye may improve the signal-to-noise ratio.

Based on combined YOYO-1 assays from all experiments, we estimate that the amount of tethered primer was 780 $\pm 30 \mathrm{fmol} / \mathrm{well}(n=72)$, and the density of primers on the well surface was 14 fmol (or $8 \times 10^{9}$ molecules) $/ \mathrm{mm}^{2}$. Coupled with the SP-PCR yields obtained above, these data show that $20 \%$, or 1 in 5 , of the covalently bound primers was extended during SP-PCR. This result is a substantial improvement over estimates of SP-PCR efficiency using other approaches (e.g., 1 in 300 primers extended at an equivalent density on a glass surface in Reference 1).

\section{$5^{\prime}$ HEG versus (dT) 10 Spacers}

We compared the SP-PCR yields from wells that had been tethered with

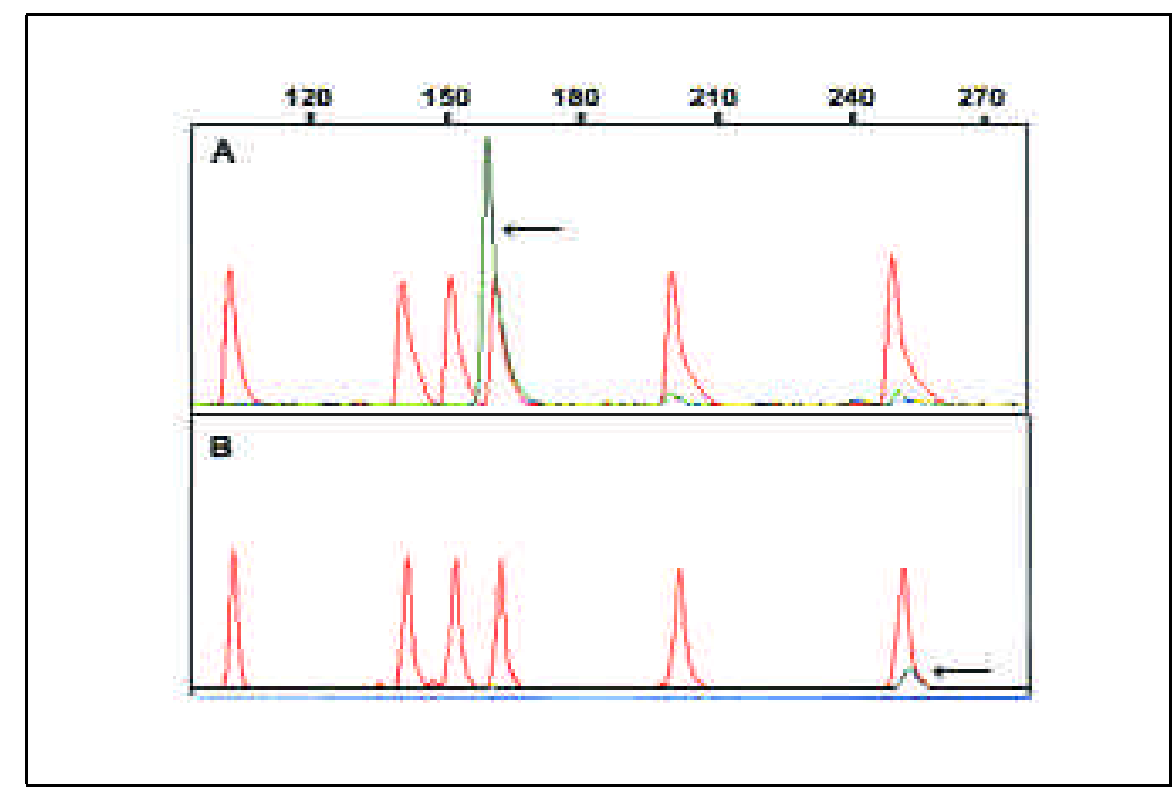

Figure 5. Electropherogram results showing the HpaII restriction fragment from SP-PCR and residual full-length product from a liquid-phase PCR "control". Top scale is size in base pairs. Color coding denotes internal size standards (red peaks) and fluorescein-labeled products (green peaks). (A) Experimental well containing tethered $\mathrm{F}^{(\mathrm{HEG})}{ }_{5}$ oligonucleotide, Taq DNA polymerase, and all other PCR components. Arrow denotes the 161-bp HpaII restriction fragment from cleavage of double-stranded SPPCR products. (B) Liquid-phase PCR control well containing all reactants except tethered oligonucleotides. Arrow indicates a weak signal at $251 \mathrm{bp}$ representing residual, full-length, liquid-phase PCR product (not cleaved by HpaII). 
$\mathrm{F}^{(\mathrm{dT})}{ }_{10}$ and $\mathrm{F}^{(\mathrm{HEG})_{5}}$ oligonucleotides. The $(\mathrm{HEG})_{5}$ spacer resulted in 2-fold more fluorescence than the $(\mathrm{dT})_{10}$ spacer, which in turn was only approximately $150 \%$ of the background (no primer) value (Table 3). Statistical analysis indicated that there were significant differences among the blank, $(\mathrm{dT})_{10}$, and $(\mathrm{HEG})_{5}$ treatments $(P<$ $0.001)$. ANOVA results showed that the $(\mathrm{HEG})_{5}$ spacer resulted in significantly greater yield than the $(\mathrm{dT})_{10}$ spacer $(P$ $<0.0001)$ and that the $(\mathrm{dT})_{10}$ spacer had significantly higher yield than the blank $(P<0.0001)$.

\section{CONCLUSION}

In general, the surface density of tethered oligonucleotides, the abundance and accessibility of complementary template molecules in solution, and the accessibility of tethered primers to Taq DNA polymerase will affect the efficiency of SP-PCR $(1,6)$. Since SP-PCR products are usually detected by enzymatic reactions that are sensitive to less than 1 amol product/ $20-\mu \mathrm{L}$ volume (9), enzyme-based assays succeed if only a small proportion of tethered oligonucleotides are extended during SP-PCR, or if residual liquidphase products remain in reaction wells. Although enzymatic detection can be done in high-throughput format, it is not quantitative and requires multiple handling steps, chemically modified probes, and expensive substrates. Here, we have demonstrated that direct fluorescent detection of SP-PCR products is feasible in NucleoLink strips. Similar results are to be expected using other commercial strips or plates as long as the tethering chemistry results in $5^{\prime}$ immobilization of sufficient quantities of oligonucleotides on the well surface. SP-PCR yields from tethered oligonucleotides with 5' (HEG) 5 spacers are significantly higher than yields from oligonucleotides with $5^{\prime}(\mathrm{dT})_{10}$ spacers. Our protocol results in a 60fold increase in extension of tethered oligonucleotides relative to reported values (1). Thus, direct detection of solid-phase amplification products should now provide a simple, quantitative, and cost-effective means of sample analysis in a variety of molecular applications.

\section{ACKNOWLEDGMENTS}

We thank Bob Sherwood of the Cornell University Biotechnology Resource Center for help with oligonucleotide synthesis. This work was funded by Cornell University, Center for Advanced Technology, and Corning, Inc.

\section{REFERENCES}

1.Adessi, C., G. Matton, G. Ayala, G. Turcatti, J.-J. Mermod, P. Mayer, and E. Kawashima. 2000. Solid phase DNA amplification: characterization of primer attachment and amplification mechanisms. Nucleic Acids Res. 28:87e.

2.Andreadis, J.D. and L.A. Chrisey. 2000. Use of immobilized PCR primers to generate covalently immobilized DNAs for in vitro transcription/translation reactions. Nucleic Acids Res. 28:e5

3.Box, G.E.P. and D.R. Cox. 1964. An analysis of transformations. J. R. Stat. Soc. Ser. B 26:211-243.

4.Cowl, J.S., N. Hartley, D.X. Xie, G.C. Whitelam, G.P. Murphy, and N.P. Harbern. 1994. The Phy Cgene of Arabidopsis: absence of the third intron found in PhyA and PhyB. Plant Physiol. 106:813-814.

5.Doyle, J.J. and J.L. Doyle. 1987. A rapid DNA isolation procedure for small amounts of leaf tissue. Phytochemical Bull. 19:11-15.

6.Guo, Z., R.A. Guifoyle, A.J. Thiel, R. Wang, and L.M. Smith. 1994. Direct fluorescence analysis of genetic polymorphisms by hybridization with oligonucleotide arrays on glass supports. Nucleic Acids Res. 22:54565465.

7.Keller, C., K. Koo, M. Mitsuhashi, and M. Ogura. 1994. Use of the fluorescent dye YOYO-1 to quantify oligonucleotides immobilized on plastic plates. BioTechniques 16:1032-1034.

8. Koch, T., N. Jacobsen, J. Fensholdt, U. Boas, M. Fenger, and M.H. Jakobsen. 2000. Photochemical Immobilization of anthraquinone conjugated oligonucleotides and PCR amplicons on solid surfaces. Bioconjugate Chem. $11: 474-483$.

9.Kohsaka, H. and D.A. Carson. 1994. Solidphase polymerase chain reaction. J. Clin. Lab. Anal. 8:452-455.

10.Oroskar, A.A., S.-E. Rasmussen, H.N. Rasmussen, S.R. Rasmussen, B.M. Sullivan, and A. Johansson. 1996. Detection of immobilized amplicons by ELISA-like techniques. Clin. Chem. 42:1547-1555.

11.Rasmussen, S.R., H.B. Rasmussen, M.R. Larsen, R. Hoff-Jørgensen, and R.J. Cano. 1994. Combined polymerase chain reactionhybridization microplate assay used to detect bovine leukemia virus and Salmonella. Clin. Chem. 40:200-205.

12.Shchepinov, M.S., S.C. Case-Green, and E.M. Southern. 1997. Steric factors influencing hybridization of nucleic acids to oligonucleotide arrays. Nucleic Acids Res. 25:1155-
1161.

13.Sjöroos, M., J. Ilonen, and T. Lövgren. 2001. Solid-phase PCR with hybridization and time-resolved fluorometry for detection of HLA-B27. Clin. Chem. 47:498-504.

14.Sokal, R.R. and F.J. Rohlf. 1995. Biometry. W.H. Freeman \& Co., New York.

15.Yang, M., R.C.Y. Kong, N. Kazmi, and A.K.C. Leung. 1998. Covalent immobilization of oligonucleotides on modified glass/silicon surfaces for solid-phase DNA hybridization and amplification. Chem. Lett. (Jpn.) 3:257-258.

Received 7 May 2001; accepted 31 August 2001.

Address correspondence to:

Dr. Stephen Kresovich

Institute for Genomic Diversity

158 Biotechnology Building

Cornell University

Ithaca, NY 14853-2703, USA

e-mail: sk20@cornell.edu

For reprints of this or any other article, contact Reprints@BioTechniques.com 